\title{
Effect of Internet Addiction on Cognition - An Event Related Potential (P300) Study
}

\author{
Ghanshyam Mishra ${ }^{1}$, Manish Gupta ${ }^{2, *}$, Neelam Vaney ${ }^{1}$
}

\section{Ghanshyam Mishra', Manish Gupta ${ }^{2, *}$, Neelam Vaney ${ }^{1}$}

'Department of Physiology, UCMS, Delhi, INDIA.

${ }^{2}$ Department of Physiology, University College of Medical Sciences and Guru Teg Bahadur Hospital, Dilshad Garden, Delhi, INDIA.

\section{*Correspondence}

\section{Dr. Manish Gupta}

Professor, Department of Physiology, University College of Medical Sciences and Guru Teg Bahadur Hospital, Dilshad Garden, Delhi-110095, INDIA.

Phone: +91 9810571981

Email: manishdrucms@gmail.com

History

- Submission Date: 12-07-2021;

- Review completed: 31-08-2021.

- Accepted Date: 21-09-2021.

DOI : 10.5530/ijcep.2021.8.3.29

Article Available online

http://www.ijcep.org/v8/i3

\section{Copyright}

(c) 2021 Phcog.Net. This is an openaccess article distributed under the terms of the Creative Commons Attribution 4.0 International license.

\begin{abstract}
Background and Aim: Younger population is more vulnerable for developing internet addiction without being even aware of it. Event related potentials alteration, indicative of abnormality of cognitive function; occur in various substance addictions. Could internet addiction contribute to these similar alterations? To address this question, we compared event related potentials in students having internet addiction to those with no internet addiction. Methods: A total of 312 medical college students were screened for internet addiction using Young Internet addiction test (IAT) questionnaire. P300 recordings (a component of event related potential) were made using EMG/NCS/EP - 4 Channel system- Octopus 4 machine and compared between 30 moderate internet addicts (IAT score $>60$ ) and 30 non addicts (IAT score $<30$ ) using independent sample $t$ test. Correlation analysis was done using Pearson correlation. The level of significance was kept at $\mathrm{P}<0.05$ for all the tests. Results: The prevalence of internet addiction was $25.3 \%$ and $10.25 \%$ for mild and moderate internet addictions respectively. No student had severe internet addiction. Moderate internet addicts spent more than twice the average weekly hours of internet usage than non addicts. P300 latency was significantly prolonged in internet addicts compared to non addicts and the amplitude of P300 wave was found to be significantly lower in internet addicts. Conclusion: The significantly increased P300 latency and reduced P300 amplitude indicates cognitive decline in internet addicts.
\end{abstract}

Key words: Cognition, Event related potential, Internet addiction, P300, Young internet addiction test.

\section{INTRODUCTION}

India has more than 627 million users of internet and $50 \%$ of them are under the age of 25 years. ${ }^{[1,2]}$ Students are considered as vulnerable population for development of various addictions. ${ }^{[3]}$ Exponential increase in internet usage is attributed to large scale availability of affordable smartphones, high speed internet data, mobile games, apps as well as social networking sites over the past 4-5 years. Young individuals apart from their normal internet usage also use internet excessively to overcome their loneliness, recreation activities and interpersonal problems.

The term internet addiction (IA) was coined by Young and she described it as excessive, problematic maladaptive preoccupation and irresistible internet use for periods of time longer than intended with significant distress or impairment resulting from the behavior and is considered as addictive disorder. ${ }^{[4]}$ For diagnosing internet addiction she developed most widely accepted questionnaire "Young's Internet Addiction Test (IAT)" according to the diagnostic criteria of the Diagnostic and Statistical manual of Mental Disorders (DSM-IV) for pathological gambling, and it was adapted for the diagnosis of
IA. ${ }^{[5]}$ Young's IAT has been validated in various languages. The IAT is in the form of a questionnaire consisting of 20 items measured with four-points ranging from "never" to "always" and accordingly each item is given a score from 0 to 5 .

Prevalence of internet addiction as seen on literature survey in Indian student population varies between 9\%-35\%. ${ }^{[6-9]}$ Behavioral addiction clinic at AIIMS, New Delhi has witnessed two fold increase in the number of people visiting this clinic for internet addiction. ${ }^{[10]}$ Internet addiction adversely affects health as it is linked with the development of disturbed circadian rhythm, reduced sleep time, disrupted sleep wake cycle, anxiety and depression. ${ }^{[11-13]}$ Study on internet addicts have shown impaired cognition, behavioral control and structural changes in the brain along with dysfunction in the brain dopaminergic systems as seen in substance addictions. ${ }^{[14]}$ These abnormalities of sleep, anxiety, depression along with dysfunction of brain systems may lead to changes in cognitive functions.
Cite this article: Mishra G, Gupta M, Vaney N. Effect of Internet Addiction on Cognition - An Event Related Potential (P300) Study. Int J Clin Exp Physiol. 2021;8(3):122-5. 
Cognitive functions can be measured by P300 component of event related potential (ERP), a component of EEG which measures the brain response directly as a result of specific sensory stimulus (event). ${ }^{[15]}$ Impaired attention may be the most common clinical symptom of neuropsychiatric disorder like addiction. ${ }^{[16]}$ Few studies have reported prolonged P300 latency in Internet addicts in South Korea and China when the effect of internet addiction on cognition was assessed. ${ }^{[17-19]}$

The present study attempted to fill up this void in literature especially in students from Indian population. In this study it was hypothesized that Internet addiction may affect cognitive functions and it aimed to assess and compare cognitive function in internet addicts and non addicts by measuring P300, an event Related potential.

\section{MATERIALS AND METHODS}

Institutional Ethics Committee-Human Research (IEC-HR) clearance was obtained before starting the study. A total of 312 medical students volunteered to take part in this study and were screened for internet addiction using Young's Internet addiction test (IAT). Scores of IAT were used to allocate students into internet addicts $(n=30)$ with IAT score $>60$ and non addicts group $(n=30)$ with IAT score $<30$. Medical students in the age group of 18-25 years having normal BMI $\left(19-25 \mathrm{~kg} / \mathrm{m}^{2}\right)$, with minimum one year duration of internet usage were included in this study. Participants with IAT score between $>30$ to $<60$, history of any substance abuse, alcoholism, traumatic brain injury, or any chronic kidney or liver disease, diabetes mellitus and hearing disorder were excluded. The work process was given as a flow chart (Figure 1).

\section{Recording of P300}

P300 in both groups was recorded in the department Neurophysiology lab using EMG/NCS/EP - 4 Channel system - Octopus 4 machine by Biostar healthcare, India. Recording was done using the silver-silver chloride disk electrodes placed at standard 10-20 International system. The electrodes were placed on the vertex of skull (Reference electrode $\mathrm{C}_{\mathrm{z}}$ ), on forehead (Ground electrode $\mathrm{FP}_{\mathrm{z}}$ ), and on both mastoid process (Active electrodes A1 and A2) (Figure 2). After cleaning the skin sites with alcohol and cotton, Nuprep skin preparation gel was rubbed over

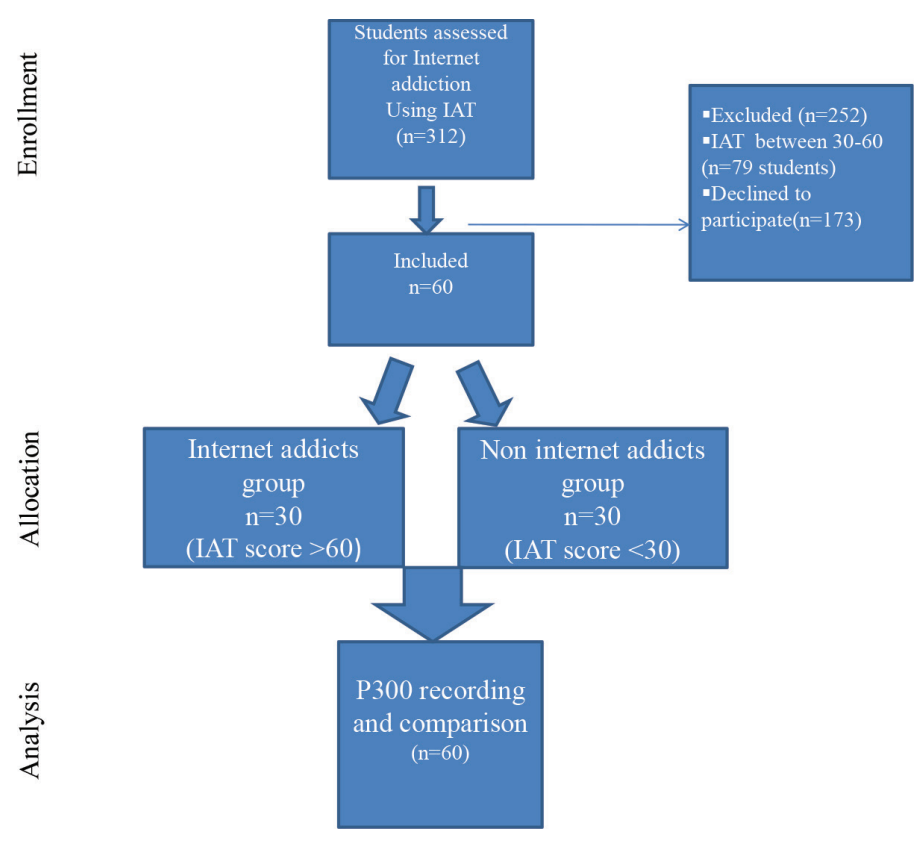

Figure 1: Workflow chart.

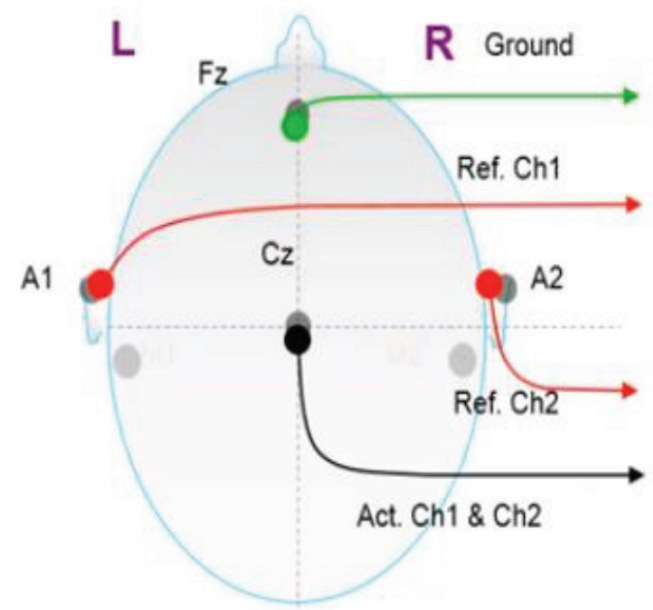

Figure 2: Site of placement of active electrode at $\mathrm{Cz}$ and reference electrodes $\mathrm{A} 1$ and $\mathrm{A} 2$ for $\mathrm{P} 300$ recording, Fz showing site for ground electrode.

the site and Ten20 conductive neurodiagnostic electrode paste was applied over clean electrodes and it was immediately fixed over the prepared 4 sites using micropore adhesive tape. Headphones were attached and response switch was given in the dominant hand of the subject. The impedance was kept below $5 \mathrm{~K} \Omega$.

To record P300 event related potentials from scalp oddball paradigm was used where two types of stimulus were given at an intensity of $90 \mathrm{~dB}$. Type 1 stimulus was a click frequency of $1000 \mathrm{~Hz}$ and $80 \%$ occurrence. A type 2 stimulus was a beep frequency of $3000 \mathrm{~Hz}$ with its occurrence $20 \%$ of the time. Subjects had to distinguish a type 2 (rare) from a type 1 (frequent) stimulus. They were asked to press a button on the response pad as quickly and accurately as possible with thumb of their dominant hand on hearing the target stimuli (auditory stimulus type 2) delivered by headphones. The subjects underwent a trial session to familiarize them with the types of stimuli and the recording procedure. The stimulus sequence was random and the response obtained was displayed on the computer screen. Peak latency of P300 and peak-to-peak amplitude of N200-P300 was recorded.

\section{Statistical Analysis of Data}

The results were expressed as mean \pm SD and were analyzed using SPSS version 20 for windows. Normally distributed data were tested with Student's unpaired $t$ test. Correlation analysis was done using Pearson correlation. The level of significance was kept at $\mathrm{P}<0.05$ for all the tests.

\section{RESULTS}

Demographic and cognitive parameters were measured in both internet addicts and non addicts groups. The prevalence of mild internet addiction was $25.3 \%$ with IAT score of $30-49$ and $10.25 \%$ with IAT score of 50-79 for moderate internet addiction. No student had severe internet addiction (Table 1).

There was no significant difference in the age of both groups. Moderate internet addicts spent more than twice the average weekly hours of internet usage ( $53 \mathrm{hr}$ ) than non addicts $(26.50 \mathrm{hr}$ ) (Table 2). Peak latency of P300 and peak-to-peak amplitude of N200-P300 was compared between internet addicts and non addicts. P300 latency was significantly prolonged in internet addicts. P300 amplitude was found to be significantly lower in internet addicts group (Table 2). Recordings of P300 of subjects who scored 27 and 62 in IAT were shown as Figure 3. 
Table 1: Prevalence of internet addiction.

\begin{tabular}{|ccc|}
\hline Internet addiction & IAT scores & Prevalence rate \\
\hline Mild & $30-49$ & $25.3 \%$ \\
Moderate & $50-79$ & $10.25 \%$ \\
Severe & $80-100$ & 0 \\
\hline
\end{tabular}

Table 2: Demographic and P300 characteristics of the participants.

$\begin{array}{cccc}\text { Variables } & \begin{array}{c}\text { Internet addicts } \\ \mathrm{n}=30\end{array} & \begin{array}{c}\text { Non addicts } \\ \mathrm{n}=30\end{array} & \text { P value } \\ \begin{array}{c}\text { Demographic data } \\ \text { Age(years) }\end{array} & 19.17 \pm 0.91 & 18.83 \pm 1.05 & 0.19 \\ \text { M/F (numbers) } & 25 / 5 & 21 / 9 & 0.22 \\ \text { Clinical data } & & & \\ \text { Duration of Internet (years) } & 5.83 \pm 1.53 & 4.77 \pm 1.77 & 0.01 \\ { }^{*} \text { Hours per week } & 53.0(28.0-60.7) & 26.50(14.0-28.5) & <0.01 \\ \text { IAT } & 66.07 \pm 5.52 & 26.83 \pm 3.83 & <0.01 \\ \text { BMI } & 21.30 \pm 1.87 & 20.50 \pm 1.65 & 0.08 \\ \text { Event Related Potential } & & & \\ \text { P300 Latency (ms) } & 270.62 \pm 31.06 & 228.23 \pm 22.32 & <0.01 \\ { }^{*} \text { N2-P300 Amplitude }(\mu \mathrm{v}) & 4.60(2.60-7.80) & 5.75(4.45-8.32) & 0.048\end{array}$

Data was presented as mean $\pm \mathrm{SD}$. ${ }^{\star}$ Values expressed as median (range)

Comparison was done using Student's unpaired $t$ test. $\mathrm{P}<0.05$ is considered statistically significant. IAT: Internet addiction test; BMI: Body mass index.

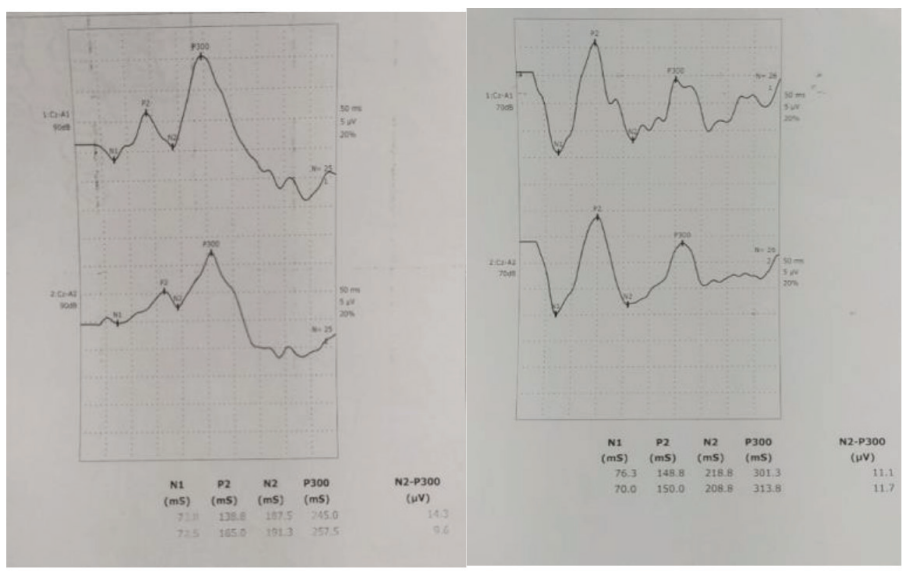

IAT score 27

IAT score 62

Figure 3: P300 of subjects scored 27 and 62 in IAT.

There was no significant correlation of IAT scores with P300 amplitude but P300 latency $(r=0.578, \mathrm{P}=0.000)$ was significantly correlated with IAT scores (Table 3).

\section{DISCUSSION}

The prevalence of internet addiction in medical students in our study is the same as shown in other Indian studies on other groups. There was significant increase in P300 latency and decrease in P300 amplitude in the internet addict group which can be well correlated with significantly
Table 3: Pearson correlation between IAT scores and P300 components.

$\begin{array}{cccc} & & \begin{array}{c}\text { P300 Latency } \\ (n=60)\end{array} & \begin{array}{c}\text { P300 Amplitude } \\ (n=60)\end{array} \\ \text { IAT scores } & \text { Pearson Correlation } & 0.578^{* *} & -0.211 \\ & \text { Sig. (2-tailed) } & 0.000 & 0.106\end{array}$

"* Correlation is significant at the 0.01 level ( 2 tailed).

higher IAT scores, duration in years and weekly hour's internet usage. These changes in P300 latency and amplitude reflect delayed information processing in addicts. This deficit in information processing may be due to slower reaction time which can be attributed to greater difficulty in discriminating between two different stimuli. Processing deficit may also be due to longer decision time or could be attributed to longer response execution time in internet addicts. Since P300 depends on contextual evaluation of stimulus, all the above factors may be contributing towards delayed P300 and its lower amplitude.

Earlier studies on mobile phone users reported that chronic mobile phone exposure does not affect cognition as no significant changes were observed in P300 latency and amplitude. ${ }^{[20]}$ Significantly altered cognitive function (increased P300 latency) in our internet addict group may again be correlated to higher IAT scores than the mobile phone users who may be non addict or having only mild internet addiction (IAT<30). Another study on internet addicts which reported increased latency of P300 in internet addicts further found that P300 latencies returned to normal levels after internet addicts completed a three-month cognitive behavioral therapy CBT program suggesting that cognitive changes were a consequence of internet addiction. ${ }^{[18]}$ Our study has also shown similar findings of significantly prolonged P300 latencies in internet addicts.

Study on internet addicts which recorded response inhibition neurologically along with event-related potentials (ERPs) concluded that internet addictsused more cognitive resources to complete the task and were less efficient at information processing. ${ }^{[21]}$ These findings are in line with our results of $\mathrm{P} 300$ on internet addicts.

Findings of another study suggest that internet addiction seems to be related with structural andprominent functional changes in cortical (e.g., prefrontal cortex and limbic structures) and subcortical (e.g., parts of the basal ganglia) brain areas. These brain changes are in turn considered as neural correlates of reductions in executive control. They introduced a cognitive-behavioral model of generalized and specific internet addiction emphasizing positive and negative reinforcement due to internet use, which lead to cue-reactivity and craving reactions. The findings of this study help us in understanding the underlying pathophysiology of internet addiction and thus explaining the mechanism behind increased P300 latency in internet addicts could be due to structural and functional changes occurring in prefrontal cortex, and limbic region of internet addicts. ${ }^{[22]}$

In a recent study on neurobiology of internet addiction, molecular mechanisms were reviewed through neuroimaging studies utilizing functional magnetic resonance imaging (fMRI), positron emission tomography (PET) and single photon emission computed tomography (SPECT) found that internet addiction is associated with dysfunction in the brain dopaminergic systems just like addiction involving substances. ${ }^{[14]}$ Similar studyrelated to the effects of internet addiction was done using fMRI and optimized voxel-based morphometry (VBM) technique, have shown changes in the microstructural integrity of major neuronal fiber pathways and microstructural changes were associated with the duration of internet addiction. These results have shown how internet addiction resulted in underlying changes in brain structure and these changes were similar to those present in substance addicts. ${ }^{[23]}$ 
Further studies on P300 in internet addiction involving more number of participants with higher IATscore $(>80)$ should be conducted for better understanding of cognitive dysfunction due to this behavioral addiction. Biochemical parameters to assess any kind of oxidative stress may also be included in future studies which we could not do due to resource constraints.

\section{CONCLUSION}

Significantly prolonged P300 latency in internet addict group of medical students leads to cognitive derangement which is positively correlated with severity of internet addiction. These P300 alterations may either be due to the structural and functional changes in the cortical, sub cortical areas or changes in the microstructural integrity of major neuronal fiber pathways or may be due to the dysfunction in the brain dopaminergic system as reported in other studies on internet addicts. Disturbed circadian rhythm, reduced sleep time,anxiety and depression reported in studies on internet addicts might also contribute to cognitive dysfunction. This decline in cognitive function can affect student's attention, learning abilities and may also hamper their abilities to acquire clinical skills must for a clinician.

\section{ACKNOWLEDGEMENTS}

We would like to thank Principal of UCMS and Head of the department of Physiology of UCMS for support and conduct of the study. We also offer our sincere thanks to the technical staff of the department of Physiology UCMS for their support.

\section{CONFLICT OF INTEREST}

The authors have declared that they have no conflict of interest.

\section{ABBREVIATIONS}

IAT: Internet Addiction Test; DSM-IV: Diagnostic and Statistical manual of Mental Disorders; ERP: Event Related Potential; fMRI: Functional Magnetic Resonance Imaging; PET: Positron Emission Tomography; SPECT: Single Photon Emission Computed Tomography; VBM: VoxelBased Morphometry.

\section{REFERENCES}

1. All India users estimates. Available from Times of India. Source IAMAI and kantar IMRBI- CUBE 207, October 2017, [updated Feb 20, 2018].

2. Nielson. 50\% of smartphone users in India are under 25 yrs. 2013 February.

3. Kandell JJ. Internet addiction on campus: the vulnerability of college students. Cyber Psychol Behav. 1998;1(1):11-7. doi: 10.1089/cpb.1998.1.11.

4. Young KS. Internet addiction: the emergence of a new clinical disorder. Cyber Psychol Behav. 1998;1(3):237-44. doi: 10.1089/cpb.1998.1.237.

5. American Psychiatric Association (APA). Diagnostic and statistical manual of mental disorders. 5th ed. Washington, DC; 2013.

6. Nath K, Naskar S, Victor R. A Cross-sectional study on the prevalence, risk factors, and ill effects of internet addiction among medical students in northeastern India. Prim Care Companion CNS Disord. 2016;18(2). doi: 10.4088/ PCC.15m01909, PMID 27486546.

7. Krishnamurthy S, Chetlapalli SK. Internet addiction: prevalence and risk factors: A cross-sectional study among college students in Bengaluru, the
Silicon Valley of India. Indian J Public Health. 2015;59(2):115-21. doi: 10.4103/0019-557X.157531, PMID 26021648.

8. I Balhara YPS, Doric A, Stevanovic D, Knez R, Singh S, Roy Chowdhury MR, et al. Correlates of problematic internet use among college and university students in eight countries: an international cross-sectional study. Asian J Psychiatr. 2019;45:113-20. doi: 10.1016/j. ajp.2019.09.004, PMID 31563832.

9. Gupta A, Khan AM, Rajoura OP, Srivastava S. Internet addiction and its mental health correlates among undergraduate college students of a university in North India. J Family Med Prim Care. 2018;7(4):721-27. doi: 10.4103/jfmpc. jfmpc_266_17, PMID 30234044.

10. Balhara YPS, Harshwardhan M, Kumar R, Singh S. Extent and pattern of problematic internet use among school students from Delhi: findings from the cyber awareness programme. Asian J Psychiatr. 2018;34:38-42. doi: 10.1016/j. ajp.2018.04.010, PMID 29631149.

11. Archana R, Sharma MK, Keshav KJ, Marimuthu P. Does mild internet use augment cognitive functions?: preliminary evidence. J Family Med Prim Care. 2019;8(12):3876-80. doi: 10.4103/jfmpc.jfmpc_488_19, PMID 31879629.

12. Reed $P$, Romano $M$, Re F, Roaro A, Osborne LA, Viganò $C$, et al. Differential physiological changes following internet exposure in higher and lower problematic internet users. PLOS ONE. 2017;12(5):e0178480. doi: 10.1371/journal. pone.0178480, PMID 28542470

13. Younes F, Halawi G, Jabbour H, El Osta NE, Karam L, Hajj A, et al. Internet addiction and relationships with insomnia, anxiety, depression, stress and self-esteem in university students: a cross-sectional designed study. PLOS ONE. 2016;11(9):e0161126. doi: 10.1371/journal. pone.0161126, PMID 27618306.

14. Zhu $Y$, Zhang $H$, Tian M. Molecular and functional imaging of internet addiction. Biomed Res Int. 2015;2015:378675. doi: 10.1155/2015/378675, PMID 25879023.

15. Bashore TR, Van der Molen MW. Discovery of the P300: A tribute. Biol Psychol. 1991;32(2-3):155-71. doi: 10.1016/0301-0511(91)90007-4, PMID 1790268.

16. Deldin PJ, Duncan CC, Miller GA. Season, gender, and P300. Biol Psychol. 1994;39(1):15-28. doi: 10.1016/0301-0511(94)90054-x, PMID 7880945.

17. Yu H, Zhao X, Li N, Wang M, Zhou P. Effect of excessive internet use on the time-frequency characteristic of EEG. Prog Nat Sci. 2009;19(10):1383-87. doi: 10.1016/j.pnsc.2008.11.015.

18. Ge L, Ge X, Xu Y, Zhang K, Zhao J, Kong X. P300 change and cognitive behavioral therapy in subjects with internet addiction disorder: A 3-month follow-up study. Neuro Reg Res. 2011;6:2037-41.

19. Zhou ZH, Yuan GZ, Yao JJ, Li C, Cheng ZH. An event-related potential investigation of eficient inhibitory control in individuals with pathological internet use. Acta Neuropsychiatr. 2010;22(5):228-36. doi: 10.1111/j.16015215.2010.00444.x, PMID 26952833.

20. Mohan M, Khaliq F, Panwar A, Vaney N. Does chronic exposure to mobile phones affect cognition? Funct Neurol. 2016;31(1):47-51. doi: 10.11138/ fneur/2016.31.1.047, PMID 27027894.

21. Zheng $H$, Hu Y, Wang $Z$, Wang $M$, Du X, Dong G. Meta-analyses of the functional neural alterations in subjects with Internet gaming disorder: similarities and differences across different paradigms. Prog Neuropsychopharmacol Biol Psychiatry. 2019;94:109656. doi: 10.1016/j.pnpbp.2019.109656.

22. Brand M, Young KS, Laier C. Prefrontal control and internet addiction: A theoretical model and review of neuropsychological and neuroimaging findings. Front Hum Neurosci. 2014;8:375. doi: 10.3389/fnhum.2014.00375, PMID 24904393.

23. Yuen EY, Wei J, Yan Z. Molecular and epigenetic mechanisms for the complex effects of stress on synaptic physiology and cognitive functions. Int J Neuropsychopharmacol. 2017;20(11):948-55. doi: 10.1093/ijnp/pyx052, PMID 29016816. 